\title{
The ATP-dependent amide ligases DdaG and DdaF assemble the fumaramoyl-dipeptide scaffold of the dapdiamide antibiotics ${ }^{\dagger}$
}

\author{
Marie A. Hollenhorst, Jon Clardy, and Christopher T. Walsh * \\ Department of Biological Chemistry and Molecular Pharmacology, Harvard Medical School, Boston, \\ MA 02115
}

\begin{abstract}
The enzymes DdaG and DdaF, encoded in the Pantoea agglomerans dapdiamide antibiotic biosynthetic gene cluster, when expressed in E. coli, 1 make the tandem amide bonds of the dapdiamide scaffold at the expense of ATP cleavage. DdaG uses fumarate, 2,3-diaminopropionate (DAP) and ATP to make fumaroyl-AMP transiently on the way to the $N_{\beta}$-fumaroyl-DAP regioisomer. Then DdaF acts as a second ATP-dependent amide ligase, but this enzyme cleaves ATP to ADP and $\mathrm{P}_{\mathrm{i}}$ during amide bond formation. However, DdaF will not accept $N_{\beta}$-fumaroyl-DAP; the enzyme requires the fumaroyl moiety to be first converted to the fumaramoyl half amide in $N_{\beta}$-fumaramoylDAP. DdaF adds Val, Ile, or Leu to the carboxylate of fumaramoyl-DAP to make the three dapdiamides A-C. Thus, to build the dapdiamide antibiotic scaffold, amidation must occur on the fumaroyl-DAP scaffold, after DdaG action but before DdaF catalysis. This is an unusual instance of two ligases acting sequentially in untemplated amide bond formations using attack of substrate carboxylates at $\mathrm{P}_{\alpha}$ (AMP-forming) and then at $\mathrm{P}_{\gamma}$ (ADP-forming) of ATP cosubstrates.
\end{abstract}

P. agglomerans strains produce a variety of antibiotics, as evidenced by the discoveries of the pantocins (1,2), andrimid (3), and most recently dapdiamides A-E (4) (Figure 1A). This family of fumaramoyl- and epoxysuccinamoyl-dipeptides is named for the presence of DAP and the two amide linkages of the antibiotic scaffold. The compounds are similar in structure to synthetic methoxyfumaroyl-DAP-dipeptides which show broad spectrum activity by targeting bacterial and fungal glucosamine-6-phosphate (GlcN-6-P) synthase, the enzyme which catalyzes the rate-limiting step in the formation of the essential cell-wall building block UDP$\mathrm{N}$-acetyl-glucosamine $(5,6)$. An analogous mode of action has been proposed for three previously isolated antibiotics with similar structures to the dapdiamides, CB-25-I from Serratia plymuthica (7) and Sch37137 (8) and A19009 $(9,10)$ from actinomycete isolates (Figure 1B) (5). These compounds are likely cleaved intracellularly to generate acyl-DAP

\footnotetext{
$\dagger$ This work was supported in part by the National Institutes of Health GM 20011 (CTW), GM 086258 (JC), and Medical Scientist Training Program GM 07753 (MAH).

${ }^{1}$ Abbreviations: P. agglomerans, Pantoea agglomerans; E. coli, Escherichia coli; ATP, adenosine-5'-triphosphate; DAP, 2,3diaminopropionate; AMP, adenosine-5'-monophosphate; ADP, adenosine-5'-diphosphate; $\mathrm{P}$, phosphate; GlcN-6-P, glucosamine-6phosphate; UDP, uridine-5'-diphosphate; NRPS, nonribosomal peptide synthetase; $\mathrm{PP}_{\mathrm{i}}$, pyrophosphate; $\mathrm{ORF}$, open reading frame; $\mathrm{PCR}$, polymerase chain reaction; Ni-NTA, nickel-nitrilotriacetic acid-agarose; SDS-PAGE, sodium dodecyl sulfate - polyacrylamide gel electrophoresis; MWCO, molecular weight cut-off; BSA, bovine serum albumin; ${ }^{1} \mathrm{H}-\mathrm{NMR}$, proton-nuclear magnetic resonance; PK, pyruvate kinase; LDH, lactate dehydrogenase; HEPES, 4-(2-hydroxyethyl)-1-piperazineethanesulfonic acid; LC, liquid chromatography; MS, mass spectrometry; Q-TOF, quadrupole time-of-flight; HPLC, high-performance liquid chromatography; LB, Luria-Bertani medium; IPTG, isopropyl- $\beta$-D-galactopyranoside; FPLC, fast protein liquid chromatography; FMOC, 9-fluorenylmethoxycarbonyl; TFA, trifluoracetic acid; $\mathrm{CH}_{3} \mathrm{CN}$, acetonitrile; DTT, dithiothreitol

*To whom correspondence should be addressed: christopher_walsh@hms.harvard.edu, phone: 617.432.1715, fax: (+1) 617.432.0483. SUPPORTING INFORMATION AVAILABLE

Figures S1-10, Tables S1-4, Schemes S1-4, and supplemental methods. This material is available free of charge via the Internet at http://pubs.acs.org.
} 
warheads which can inhibit GlcN-6-P synthase, as demonstrated for $N_{\beta}$-fumaramoyl-DAP (11) (Figure 1A). The fumaramoyl-DAP warheads derived from A19009 and dapdiamides A$\mathrm{D}$ would provide a conjugated eneamide system that is likely attacked by the thiolate side chain of the active site Cys in the glutaminase domain of GlcN-6-P synthase in analogy to the mechanism of inactivation observed for $N_{\beta}$-methoxyfumaroyl-DAP (5) (Figure 1C). Alkylation of this residue blocks generation of the ammonia required to convert fructose-6-P into glucosamine-6-P. The epoxysuccinamoyl moiety in CB-25-I, Sch37137 and dapdiamide E could likewise function as an inactivating electrophile for the catalytic Cys in the target enzyme.

Peptide antibiotics are typically formed by two biological strategies. The first involves ribosomal synthesis of protein precursors that undergo posttranslational proteolysis and/or modification and is exemplified by the Microcin B17, C7 and E492 molecules as well as the lantibiotics such as nisin, and the thiazolyl peptides such as thiostrepton and thiocillins (12). The second strategy uses nonribosomal peptide synthetase (NRPS) assembly lines to specify the order and composition of amino acid monomers incorporated and accounts for the biogenesis of penicillin, vancomycin, and daptomycin scaffolds among others (12). However, there is another way that peptides can be assembled, independent of ribosomal-based mRNA or NRPS machinery, via ATP-dependent coupling of amino acid monomers and elongating peptides by soluble enzymes. This route has precedents in glutathione biosynthesis (13) and bacterial cell wall pentapeptide elongation (14) where ATP is cleaved to ADP and intermediate acyl phosphates are formed as reactive species. A variant of this route occurs in the ATPdependent elongation and cyclization of the siderophore ferrioxamine $\mathrm{E}$ (15) by cleavage of ATP to AMP and $\mathrm{PP}_{\mathrm{i}}$ and involves acyl-AMP intermediates. We have found that the acyl dipeptides of the dapdiamide antibiotic family are constructed by this third strategy, via tandem action of two soluble ligases, one (DdaG) that cleaves ATP to AMP and another (DdaF) that cleaves ATP to ADP.

While no biosynthetic gene information is available for CB-25-I, Sch37137 or A19009, a single gene cluster from $P$. agglomerans that makes all five dapdiamides has recently been cloned into E. coli (4) (Figure S1). This metabolic capacity from one gene cluster suggests both pathway enzyme promiscuity and the prospect of increased scaffold diversity from combinatorial biosynthesis once the catalysts have been identified. Inspection of the fumaramoyl/epoxysuccinamoyl-dipeptide scaffold of the dapdiamides and the encoding biosynthetic gene cluster has led to predictions about the possible functions of the encoded ORFs (4). Of particular importance here are DdaG and DdaF, which are predicted to be ATPdependent amide ligases and thus are candidates for making the two peptide bonds.

Intriguingly, DdaG has the signature elements of an adenylating ligase that cleaves ATP to AMP and $\mathrm{PP}_{\mathrm{i}}$, while DdaF is predicted to be an ATP grasp family member (16) and instead cleave ATP to ADP and $\mathrm{P}_{\mathrm{i}}$ in a phosphoryl transfer mechanism.

In this study we report heterologous expression, purification, and characterization of DdaG and DdaF and their amide ligase activities for making $N_{\beta}$-fumaroyl-DAP and $N_{\beta}$-fumaramoylDAP-Val/Ile/Leu, respectively.

\section{MATERIALS AND METHODS}

\section{Bacterial Strains, Plasmids, Materials, and Instrumentation}

Oligonucleotide primers were synthesized by Integrated DNA Technologies (Coralville, IA). PCR was performed with Phusion High-Fidelity PCR Mastermix (New England Biolabs). Cloning was performed using the Gateway System (Invitrogen). One Shot Chemically Competent TOP10 E. coli (Invitrogen) and NovaBlue(DE3) (Novagen) were used for routine cloning and propagation of DNA vectors. Recombinant plasmid DNA was purified with a 
Qiaprep kit (Qiagen). DNA sequencing was performed at the Molecular Biology Core Facilities of the Dana Farber Cancer Institute (Boston, MA). Nickel-nitrilotriacetic acid-agarose (NiNTA) superflow resin and SDS-PAGE gels were purchased from Qiagen. Protein samples were concentrated using $30 \mathrm{kDa}$ MWCO Amicon Ultra filters (Millipore). Protein concentrations were determined by Bradford assay with BSA as a standard. Chemicals were purchased from Sigma-Aldrich. NMR solvents were purchased from Cambridge Isotopes.

A pyruvate kinase/lactate dehydrogenase (PK/LDH) enzyme mix from rabbit muscle was purchased from Sigma as a buffered aqueous glycerol solution. Myokinase from chicken muscle was purchased from Sigma as a lyophilized powder and resuspended in $10 \mathrm{mM}$ HEPES, $\mathrm{pH}$ 8. Synthetic dapdiamide A and the plasmid containing the dapdiamide gene cluster, pUC19 A10A, were provided by Jessica Dawlaty. Fumaramic acid was prepared from monomethyl fumarate as described previously (17) or from monoethyl fumarate via an analogous procedure.

${ }^{1} \mathrm{H}-\mathrm{NMR}$ spectra were recorded on Varian 400 or $600 \mathrm{MHz}$ spectrometers. MS analysis was performed on an Agilent Technologies 6520 Accurate-Mass Q-TOF LC/MS, an Agilent Technologies 6210 Accurate-Mass TOF LC/MS, or by staff at the Harvard University Mass Spectrometry Laboratory (Cambridge, MA). HPLC data was collected on a Beckman Coulter System Gold (126 solvent module, 168 detector). An Alltech Alltima C18 $(250 \times 4.6 \mathrm{~mm})$ column was used for routine analytical HPLC. A Chiralcel OD-RH $(150 \times 4.6 \mathrm{~mm})$ chiral column was used for separation of FMOC-derivatized $N$-fumaroyl-DAP regioisomers. A Phenomenex Luna C18 $(250 \times 21.2 \mathrm{~mm})$ column was used for preparative HPLC.

\section{Cloning, Overexpression, and Purification of DdaG and DdaF}

$d d a G$ and $d d a F$ were PCR amplified from pUC19 A10A. For $d d a G$, the forward primer was 5'-CACCAATAAGGGAGAAAACATGAATCTGGAAATG-3' and the reverse primer was 5'-TTAATAGCGGCGTTTACTGACGTTGCCG-3'. For $d d a F$, the forward primer was 5'CACCTCGATTTTGAACAATAAAGAAGTCATCGTAATC- $3^{\prime}$ and the reverse primer was 5'-TTATTCATCAATTAGGCCCAAGTGATAAAAATCCG-3'. Unpurified PCR products were mixed with the pENTR/D-TOPO vector and the TOPO construct was then transformed into TOP10 E. coli. Purified vector was analyzed by PCR to confirm the presence of insert. The insert was recombined into pDEST17, and the product of this reaction was transformed into TOP10 E. coli. Purified vector was sequenced to confirm the insert. BL21 (DE3) E. coli were transformed with pDEST17-ddaF or pDEST17-ddaG. $25 \mathrm{~mL}$ cultures were grown overnight at $37^{\circ} \mathrm{C}$ in Luria-Bertani (LB) medium supplemented with $50 \mu \mathrm{g} / \mathrm{mL}$ carbenicillin. $2 \mathrm{~L} \mathrm{LB}$ with $50 \mu \mathrm{g} / \mathrm{mL}$ carbenicillin was inoculated with $10 \mathrm{~mL}$ of this overnight culture. The cultures were incubated at $37^{\circ} \mathrm{C}$ for $3.5-5$ hours, then $15^{\circ} \mathrm{C}$ for $0.5-1$ hour. Cultures were induced with $0.5 \mathrm{mM}$ IPTG. The cultures were incubated at $15^{\circ} \mathrm{C}$ for 20 hours, then the cells were harvested by centrifugation at $4200 \mathrm{rpm}$ for 12 minutes. Cell pellets were resuspended in $10 \mathrm{~mL}$ lysis buffer ( $10 \mathrm{mM}$ Tris. $\mathrm{HCl}, \mathrm{pH} 8,200 \mathrm{mM} \mathrm{NaCl}$ or $10 \mathrm{mM}$ HEPES, pH 8, 200 $\mathrm{mM} \mathrm{NaCl}$ ) and lysed at 5,000-10,000 psi in an Avestin EmulsiFlex-C5 high-pressure homogenizer. The cell debris was removed by ultracentrifugation at 35,000 rpm for 35 minutes. Supernatants were applied to a low pressure chromatography column containing Ni-NTA resin pre-equilibrated in lysis buffer. The resin was washed with lysis buffer containing $20 \mathrm{mM}$ imidazole. The protein was eluted with lysis buffer containing $80 \mathrm{mM}$ and $200 \mathrm{mM}$ imidazole. Protein was concentrated and imidazole removed using $30 \mathrm{kDa}$ MWCO tubes. In some cases additional purification was achieved by gel filtration chromatography on an Amersham Pharmacia Biotech AKTA FPLC using a Superdex 75 26/60 Hiload column. Aliquots of the proteins were flash-frozen in liquid nitrogen and stored at $-80{ }^{\circ} \mathrm{C}$. 


\section{DdaG and DdaF HPLC activity assays}

$50 \mu \mathrm{L}$ reactions were incubated at room temperature and contained $9.5 \mu \mathrm{M}$ enzyme, 2.5 or 5 $\mathrm{mM}$ amine substrate, 2.5 or $5 \mathrm{mM}$ carboxylate substrate, $6 \mathrm{mM}$ ATP, $10 \mathrm{mM} \mathrm{MgCl}_{2}$, and 100 $\mathrm{mM}$ HEPES, $\mathrm{pH}$ 8. Protein was removed by centrifugation in $5 \mathrm{kDa}$ MWCO filter tubes at $12,000 \times \mathrm{g}$ for 15 minutes, $100 \mu \mathrm{L}$ water was added, then filter tubes were spun again at 12,000 $\times$ g for 15 minutes. $80 \mu \mathrm{L}$ of filtered reaction mixture was combined with $80 \mu \mathrm{L} \mathrm{CH}_{3} \mathrm{CN}, 50$ $\mu \mathrm{L}$ water, $40 \mu \mathrm{L} 10 \mathrm{mM}$ FMOC-Cl (freshly prepared solution in $\mathrm{CH}_{3} \mathrm{CN}$ ) and incubated at room temperature for at least 5 minutes. $80 \mu \mathrm{L} 100 \mathrm{mM}$ aminoadamantane (in 1:1 $\mathrm{CH}_{3} \mathrm{CN}$ $\mathrm{H}_{2} \mathrm{O}$ ) was added and reactions incubated at room temperature for at least 5 minutes before HPLC analysis using a gradient of $20-100 \%$ solvent $\mathrm{B}\left(0.1 \%\right.$ TFA in $\left.\mathrm{CH}_{3} \mathrm{CN}\right)$ in solvent A ( $0.1 \%$ TFA in water) over 25 minutes. For resolution of $N$-fumaroyl-DAP regioisomers, FMOC-derivatized samples were analyzed by chiral HPLC using a gradient of 20-70\% solvent $\mathrm{B}\left(0.1 \%\right.$ TFA in $\left.\mathrm{CH}_{3} \mathrm{CN}\right)$ in solvent $\mathrm{A}(0.1 \% \mathrm{TFA}$ in water) over 35 minutes. The absorbance was monitored at $263 \mathrm{~nm}$.

\section{Coupled Spectrophotometric Assays for AMP or ADP production}

For all experiments, $200 \mu \mathrm{L}$ reactions were incubated at room temperature and contained 10 mM ATP, $12 \mathrm{mM} \mathrm{MgCl} 2,200 \mu \mathrm{M}$ NADH, $500 \mu \mathrm{M}$ phosphoenolpyruvate, and $100 \mathrm{mM}$ HEPES, pH 8. For characterization of DdaG, reactions additionally contained 43 units $/ \mathrm{mL}$ LDH, 30 units $/ \mathrm{mL} \mathrm{PK,} 21$ units $/ \mathrm{mL}$ myokinase, $10 \mathrm{mM}$ DAP, and $1.9 \mathrm{nM} \mathrm{DdaG} \mathrm{for} \mathrm{fumarate}$ assays or $9.5 \mathrm{nM}$ DdaG for succinate assays. For comparison of $N_{\alpha^{-}}$and $N_{\beta^{-}}$-fumar(am)oylDAP substrates for DdaF, reactions additionally contained 30 units $/ \mathrm{mL} \mathrm{LDH,} 15$ units $/ \mathrm{mL}$ PK, $2 \mathrm{mM} \mathrm{Val}$, and $240 \mathrm{nM}$ DdaF. For determination of the DdaF reaction velocity with varying concentrations of $N_{\beta}$-fumaramoyl-DAP, reactions additionally contained 60 units $/ \mathrm{mL} \mathrm{LDH,}$ 42 units/mL PK, $240 \mathrm{nM}$ DdaF, and $5 \mathrm{mM}$ Val. For characterization of DdaF with respect to

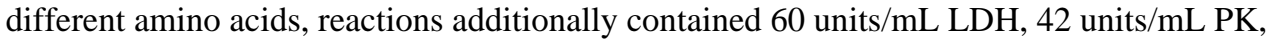
$125 \mu \mathrm{M} \mathrm{N} N_{\beta}$-fumaramoyl-DAP, and $240 \mathrm{nM}$ DdaF. The consumption of NADH was monitored continuously in a Molecular Devices SPECTRAmax PLUS 96-well plate reader by measuring the absorbance at $340 \mathrm{~nm}$ every 20 seconds. Kinetic constants were derived from velocity vs. substrate concentration data using a non-linear, least squares fitting method with GraphPad Prism software.

\section{$\mathrm{ATP} /\left[{ }^{32} \mathrm{P}\right]-\mathrm{PP}_{\mathrm{i}}$ Exchange Assays}

$1.2 \mathrm{~mL}$ reactions contained $1.9 \mu \mathrm{M} \mathrm{DdaG}, 5 \mathrm{mM}$ substrate, $1 \mathrm{mM} \mathrm{ATP}, 1 \mathrm{mM} \mathrm{MgCl}_{2}, 40 \mathrm{mM}$ $\mathrm{KCl}, 1 \mathrm{mMDTT}, 5 \mathrm{mM} \mathrm{Na}\left[{ }^{32} \mathrm{P}\right]-\mathrm{PP}_{\mathrm{i}}\left(3.3 \times 10^{4} \mathrm{cpm} / \mathrm{mL}\right)$, and $50 \mathrm{mM}$ Tris $\cdot \mathrm{HCl}, \mathrm{pH}$ 8. Reactions were incubated at $37^{\circ} \mathrm{C}$ for 30 minutes and then $350 \mu \mathrm{L}$ aliquots were removed and quenched with $750 \mu \mathrm{L}$ charcoal suspension $\left(100 \mathrm{mM} \mathrm{NaPP}_{\mathrm{i}}, 350 \mathrm{mM} \mathrm{HClO}_{4}, 16 \mathrm{~g} / \mathrm{L}\right.$ charcoal). The mixtures were vortexed, then centrifuged at $13,000 \mathrm{rpm}$ for 3 minutes. The pellets were washed twice with $750 \mu \mathrm{L}$ wash solution $\left(100 \mathrm{mM} \mathrm{NaPP}_{\mathrm{i}}, 350 \mathrm{mM} \mathrm{HClO}_{4}\right)$. Charcoal-bound radioactivity was measured on a Beckman LS 6500 scintillation counter.

\section{RESULTS}

\section{Expression and Purification of DdaG and DdaF in E. coli}

$d d a G$ and $d d a F$ were PCR amplified from pUC19 A10A, a plasmid containing the dapdiamide gene cluster, and cloned into an expression vector encoding an $\mathrm{N}$-terminal $\mathrm{His}_{6}$ tag. The proteins were overexpressed in E. coli BL21 (DE3) and purified by Ni-NTA affinity chromatography (Figure S2). Yields ranged from $12-16 \mathrm{mg} / \mathrm{L}$ for DdaG and 6-11 mg/L for DdaF. 


\section{DdaG is a Regioselective ATP-dependent Fumaroyl-DAP Ligase}

A small amount of $N_{\beta}$-fumaramoyl-DAP was isolated with the dapdiamides from fermentation (4), suggesting that acylation of DAP precedes the formation of the DAP-amino acid amide bond. To determine which enzyme is responsible for this acylating activity, purified DdaG and DdaF were incubated with DAP and potential carboxylate substrates, and reaction mixtures were derivatized with FMOC and analyzed by HPLC.

DdaG ligates fumarate and DAP to form $N$-fumaroyl-DAP in an ATP-dependent manner (Figure 2A and Table S1). The enzyme also forms $N$-succinoyl-DAP from succinate and DAP (Figure S3 and Table S1). In contrast, the ligase showed no activity in these assays with the corresponding half-amides, fumaramate and succinamate, or with the two enantiomers of trans-epoxysuccinate (Figure S4). A coupled spectrophotometric assay for AMP formation was used to kinetically characterize DdaG, and these experiments demonstrated that the enzyme is specific for fumarate over succinate, with a $k_{c a t} / K_{\mathrm{m}}$ of $21 \mathrm{mM}^{-1} \mathrm{~s}^{-1}$ for fumarate and $0.19 \mathrm{mM}^{-1} \mathrm{~s}^{-1}$ for succinate (Table S2).

$\mathrm{ATP} /\left[{ }^{32} \mathrm{P}\right]-\mathrm{PP}_{\mathrm{i}}$ exchange assays, which test for the reversible formation of adenylated intermediates, demonstrated that DdaG adenylates its carboxylate substrate as predicted based on its homology to AMP-forming enzymes (Figure 2B). As expected, activation of both fumarate and succinate was observed, whereas there was no activation of either the epoxysuccinates or succinamate. Exchange was seen with fumaramate as a result of contamination of this substrate with fumarate. ${ }^{2}$

In dapdiamides A-C, fumaramate and DAP are linked through the DAP $\beta$-amino group, while in dapdiamides $\mathrm{D}$ and $\mathrm{E}$, it is the $\alpha$-amino group that is acylated. This suggested that $\mathrm{DdaG}$ could act in a regiopromiscuous manner, accepting either the DAP $\alpha$ or $\beta$ amine as a nucleophile. To test this hypothesis, both fumaroyl-DAP isomers were synthesized (Scheme S1 and S2) and their FMOC derivatives separated by chiral HPLC. The FMOC-derivatized product of DdaG catalysis coeluted exclusively with the $\beta$ isomer, demonstrating that DdaG acts in a regioselective manner to form $N_{\beta}$-fumaroyl-DAP exclusively (Figure 2C).

\section{DdaF is an ATP-dependent $\boldsymbol{N}_{\boldsymbol{\beta}}$-fumaramoyl-DAP-amino acid ligase}

DdaF was characterized using both an ADP production assay and the same HPLC assay used to study DdaG. These experiments showed that DdaF catalyzes the second subunit condensation reaction in this pathway. The enzyme is not active with either the product of DdaG catalysis, $N_{\beta}$-fumaroyl-DAP, or the corresponding $\alpha$ isomer (Figure 3 and S5). DdaF accepts only the corresponding amide $N_{\beta}$-fumaramoyl-DAP, which it can ligate to Val, Ile, and Leu to form dapdiamides A-C (Figure 3, 4, S6, and Table S3). DdaF is specific for the $N_{\beta}$-isomer of fumaroyl-DAP; it will not ligate $N_{\alpha}$-fumaramoyl-DAP to Val (Figure 3 and S7).

Apparent substrate inhibition was observed at $N_{\beta}$-fumaramoyl-DAP concentrations above 500 $\mu \mathrm{M}$ (Figure S8), so kinetics with respect to the three amino acid substrates were determined at a fixed $N_{\beta}$-fumaramoyl-DAP concentration of $125 \mu \mathrm{M}$. While the apparent $k_{c a t}$ values for each amino acid were similar, the apparent $K_{\mathrm{m}}$ values were $260 \mu \mathrm{M}$ for Val, $830 \mu \mathrm{M}$ for Ile, and $7.82 \mathrm{mM}$ for Leu (Table S4).

\footnotetext{
${ }^{2}$ Fumaramate used in this assay was prepared from commercial monoethyl fumarate that is contaminated with a small amount of fumarate. Kinetic analysis of the contaminated fumaramate is consistent with the hypothesis that DdaG activity with this substrate results from contamination with $2-5 \%$ fumarate.
} 


\section{Stability of the $N_{\beta}$-acyl-DAP Compounds}

The lack of DdaF activity with $N_{\alpha}$-fumaramoyl-DAP makes the origin of the $\alpha$-linked dapdiamides D and E mysterious. We suspected that these compounds could arise from nonenzymatic isomerization of the corresponding $\beta$-linked regioisomers, so we tested the stability of dapdiamide $\mathrm{A}$ in $500 \mathrm{mM} \mathrm{NH}_{4} \mathrm{OH}$ at room temperature overnight. However, we did not observe any isomerization (Figure S9). We also tested for possible isomerization of the pathway intermediates. There was no conversion of $N_{\beta}$-fumaroyl-DAP to the $\alpha$-regioisomer when it was incubated at $\mathrm{pH} 8$ for three days (Figure S10). Preliminary results indicate that isomerization of the corresponding amide, $N_{\beta}$-fumaramoyl-DAP, also does not happen readily at $\mathrm{pH}$ 9.5. On the other hand, some frozen samples of $N_{\alpha}$-fumaramoyl-DAP (but not the $\mathrm{N}_{\beta}$ isomer) decomposed but the process has not been examined thoroughly.

\section{DISCUSSION}

We observe that DdaG uses fumarate, DAP, and ATP to generate $N_{\beta}$-fumaroyl-DAP as ATP is cleaved to AMP and $\mathrm{PP}_{\mathrm{i}}$. DAP can in principle be used as a nucleophile either at the $\beta$ $\mathrm{NH}_{2}$ (on the way to dapdiamides A-C) or at the $\alpha-\mathrm{NH}_{2}$ (on the way to dapdiamides D-E). In these studies only the $\beta$ coupling regiochemistry was seen with purified DdaG. In turn DdaF cleaves ATP to ADP and $\mathrm{P}_{\mathrm{i}}$ as $N_{\beta}$-fumaramoyl-DAP is coupled to Val, Ile, or Leu. Most notably, while DdaG will not accept fumaramate in place of fumarate as coupling substrate, DdaF requires fumaramoyl-DAP rather than fumaroyl-DAP.

The characterization of DdaF and DdaG allows us to propose the timing of other catalytic events in this biosynthetic pathway. Bioinformatic analysis suggested that DdaH is an amidotransferase (4), and our results imply that this enzyme acts after DdaG, converting $N_{\beta^{-}}$ fumaroyl-DAP to $N_{\beta}$-fumaramoyl-DAP. DdaF can then ligate this compound to three branched chain amino acids (Scheme 1). DdaC is a putative Fe(II)/ $\alpha$-ketoglutarate-dependent dioxygenase and is likely responsible for the formation of the dapdiamide $\mathrm{E}$ epoxide. Because DdaG shows no activity with epoxysuccinate, it is probable that DdaC acts on a double bond of either fumar(am)oyl-DAP or fumaramoyl-DAP-Val. This epoxidation may occur while the substrate is tethered to the adenylation-thiolation enzyme $\mathrm{DdaD}$, and the oxidized substrate could be released by the thioesterase homolog DdaE. The origin of the $N_{\alpha}$-acyl-DAP linkage in dapdiamides $\mathrm{D}$ and $\mathrm{E}$ remains an open question as we have been unable to observe any $N_{\alpha^{-}}$ acyl-DAP formation either from enzymatic or nonenzymatic conversion. These compounds could potentially be formed via the action of an enzyme that catalyzes an $\alpha / \beta$ isomerization or via the action of a DAP $\alpha-\mathrm{NH}_{2}$-specific ligase, however these activities do not appear to be encoded in the dapdiamide gene cluster.

The dapdiamides are the first example of a family of small molecules targeted against GlcN-6$\mathrm{P}$ synthase that are produced by a single biosynthetic gene cluster. The production of a suite of compounds instead of a single antibiotic may allow the producing organism to target a broader range of microbes and/or blunt development of target resistance. It has been shown that the identity of the amino acid ligated to DAP in the methoxyfumaroyl-DAP-dipeptides influences the rate of target-cell uptake (6), and this is likely also true for the dapdiamides. The promiscuity of DdaF with respect to its amino acid substrate allows the formation of compounds with three different C-terminal amino acids, and each may interact differently with the nonspecific peptide permeases that import these types of compounds. Additionally, the two different electrophilic moieties (Michael acceptor or epoxide) may have different levels of reactivity with the target enzyme. It remains to be explored whether production of a family of structurally distinct compounds with the same target confers an advantage on the producer, but this may be one explanation for the evolution of substrate promiscuity in the dapdiamide pathway. 
Here we focused on the two amide-bond forming enzymes that are required to generate the fumaramoyl-DAP-dipeptide scaffold of the dapdiamides. These catalysts are new members of an expanding class of ligases that assemble antibiotic peptides in a non-ribosomal and nonNRPS-dependent manner. Such enzymes typically use one of two modes carboxylate substrate activation: adenylation or phosphorylation. Like DdaG, the amide ligases CouL, NovL, and CloL use the first strategy to activate carboxylates for nucleophilic attack by donor amino groups to bring together monomers in the assembly of the aminocoumarin antibiotics coumermycin, novobiocin, and clorobiocin, respectively (18-20). In contrast, ATP grasp family members in the bacilysin $(21,22)$ and tabtoxin $(23)$ pathways likely activate carboxylate substrates via acyl-phosphate formation, like DdaF. The dapdiamide pathway is an atypical example of the recruitment of two amide ligases with distinct ATP cleavage patterns to form tandem peptide bonds.

Recent studies of $P$. agglomerans antibiotics have led to the identification of several novel condensation catalysts (24) which are homologs of enzymes from primary metabolism, suggesting that nature's repertoire of condensation catalysts for natural product biosynthesis may be broader than once suspected. A detailed understanding of the function of these catalysts may open the door to the study of new classes of compounds that are not produced by canonical biosynthetic enzymes and that may have structural features which set them apart from previously identified natural products.

\section{Supplementary Material}

Refer to Web version on PubMed Central for supplementary material.

\section{Acknowledgments}

We thank Jessica Dawlaty for providing a sample of synthetic dapdiamide A and the pUC19 A10A plasmid containing the dapdiamide gene cluster. We thank Elizabeth Sattely, Christopher Neumann, Emily Balskus, and Michael Fischbach for helpful discussions.

\section{References}

1. Brady SF, Wright SA, Lee JC, Suton AE, Zumoff CH, Wodzinski RS, Beer SV, Clardy J. Pantocin B, an antibiotic from Erwinia herbicola discovered by heterologous expression of cloned genes. J Am Chem Soc 1999:11912-11913.

2. Jin M, Liu L, Wright SAI, Beer SV, Clardy J. Structural and functional analysis of Pantocin A: an antibiotic from Pantoea agglomerans discovered by heterologous expression of cloned genes. Angew Chem, Int Ed 2003;42:2898-2901.

3. Jin M, Fischbach MA, Clardy J. A biosynthetic gene cluster for the acetyl-CoA carboxylase inhibitor andrimid. J Am Chem Soc 2006;128:10660-10661. [PubMed: 16910643]

4. a Dawlaty, JA. PhD Thesis. Cornell University; Ithaca, NY: 2008. Bioactive natural products from Pantoea agglomerans, Janithinobacterium lividum, and Conocarpus erectus. b Dawlaty J, Fischbach MA, Clardy J. Personal communications.

5. Kucharczyk N, Denisot MA, Le Goffic F, Badet B. Glucosamine-6-phosphate synthase from Escherichia coli: determination of the mechanism of inactivation by $\mathrm{N}^{3}$-fumaroyl-L-2,3diaminopropionic derivatives. Biochemistry (Mosc) 1990;29:3668-3676.

6. Milewski S, Andruszkiewicz R, Kasprzak L, Mazerski J, Mignini F, Borowski E. Mechanism of action of anticandidal dipeptides containing inhibitors of glucosamine-6-phosphate synthase. Antimicrob Agents Chemother 1991;35:36-43. [PubMed: 1901701]

7. Shoji J, Hinoo H, Sakazaki R, Kato T, Hattori T, Matsumoto K, Tawara K, Kikuchi J, Terui Y. Isolation of CB-25-I, an antifungal antibiotic, from Serratia plymuthica. J Antibiot 1989;42:869-874. [PubMed: 2500411] 
8. Cooper R, Horan AC, Gentile F, Gullo V, Loebenberg D, Marquez J, Patel M, Puar MS, Truumees I. Sch 37137, a novel antifungal compound produced by a Micromonospora sp. Taxonomy, fermentation, isolation, structure and biological properties. J Antibiot 1988;41:13-19. [PubMed: 3346184]

9. Molloy BB, Lively DH, Gale RM, Forman M, Boeck LD, Higgens CE, Kastner RE, Huckstep LL, Neuss N. A new dipeptide antibiotic from Streptomyces collinus, Lindenbein. J Antibiot 1972;25:137140. [PubMed: 4624614]

10. van der Baan JL, Barnick JWFK, Bickelhaupt F. Antibiotic A 19009 Structural Investigation and Synthesis. J Antibiot 1983;36:784-792. [PubMed: 6885634]

11. Chmara H, Andruszkiewicz R, Borowski E. Inactivation of glucosamine-6-phosphate synthetase from Salmonella typhimurium LT2 by fumaroyl diaminopropanoic acid derivatives, a novel group of glutamine analogs. Biochim Biophys Acta 1985;870:357-366. [PubMed: 3082365]

12. Nolan EM, Walsh CT. How Nature Morphs Peptide Scaffolds into Antibiotics. Chem Bio Chem 2009;10:34-53.

13. Mooz ED, Meister A. Tripeptide (glutathione) synthetase. Purification, properties, and mechanism of action. Biochemistry (Mosc) 1967;6:1722-1734.

14. Walsh CT. Enzymes in the D-alanine branch of bacterial cell wall peptidoglycan assembly. J Biol Chem 1989;264:2393-2396. [PubMed: 2644260]

15. Kadi N, Oves-Costales D, Barona-Gomez F, Challis GL. A new family of ATP-dependent oligomerization-macrocyclization biocatalysts. Nat Chem Biol 2007;3:652-656. [PubMed: 17704771]

16. Galperin MI, Koonin EV. A diverse superfamily of enzymes with ATP-dependent carboxylate-amine/ thiol ligase activity. Protein Sci 1997;6:2639-2643. [PubMed: 9416615]

17. Talley EA, Fitzpatrick TJ, Porter WL. Formation of fumaramic acid from asparagine in phosphate buffer. J Am Chem Soc 1959;81:174-175.

18. Schmutz E, Steffensky M, Schmidt J, Porzel A, Li SM, Heide L. An unusual amide synthetase (CouL) from the coumermycin $\mathrm{A}_{1}$ biosynthetic gene cluster from Streptomyces rishiriensis DSM 40489. Eur J Biochem 2003;270:4413-4419. [PubMed: 14622269]

19. Steffensky M, Li SM, Heide L. Cloning, overexpression, and purification of novobiocic acid synthetase from Streptomyces spheroides NCIMB 11891. J Biol Chem 2000;275:21754-21760. [PubMed: 10801869]

20. Galm U, Dessoy MA, Schmidt J, Wessjohann LA, Heide L. In vitro and in vivo production of new aminocoumarins by a combined biochemical, genetic, and synthetic approach. Chem Biol 2004;11:173-183. [PubMed: 15123279]

21. Steinborn G, Hajirezaei MR, Hofemeister J. bac genes for recombinant bacilysin and anticapsin production in Bacillus host strains. Arch Microbiol 2005;183:71-79. [PubMed: 15609023]

22. Tabata K, Ikeda H, Hashimoto S-I. ywfE in Bacillus subtilis codes for a novel enzyme, L-amino acid ligase. J Bacteriol 2005;187:5195-5202. [PubMed: 16030213]

23. Kinscherf TG, Willis DK. The biosynthetic gene cluster for the beta-lactam antibiotic tabtoxin in Pseudomonas syringae. J Antibiot (Tokyo) 2005;58:817-821. [PubMed: 16506699]

24. Fortin PD, Walsh CT, Magarvey NA. A transglutaminase homologue as a condensation catalyst in antibiotic assembly lines. Nature 2007;448:824-827. [PubMed: 17653193] 


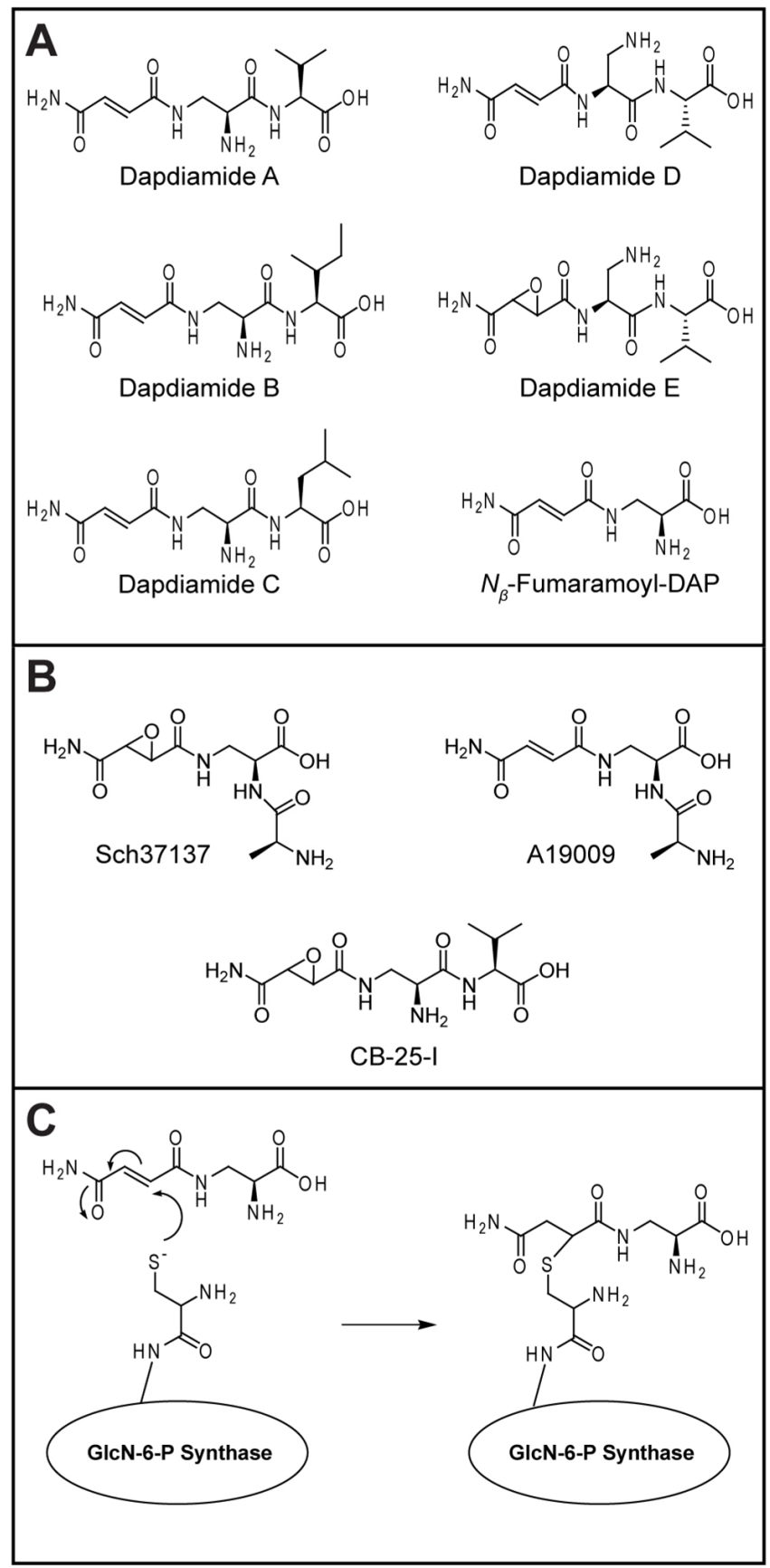

Figure 1.

A) The dapdiamide family of antibiotics and $N_{\beta}$-fumaramoyl-DAP. B) Dapdiamide-related natural products. C) Proposed mechanism of GlcN-6-P synthase inhibition by $N_{\beta}$-fumaramoylDAP. 


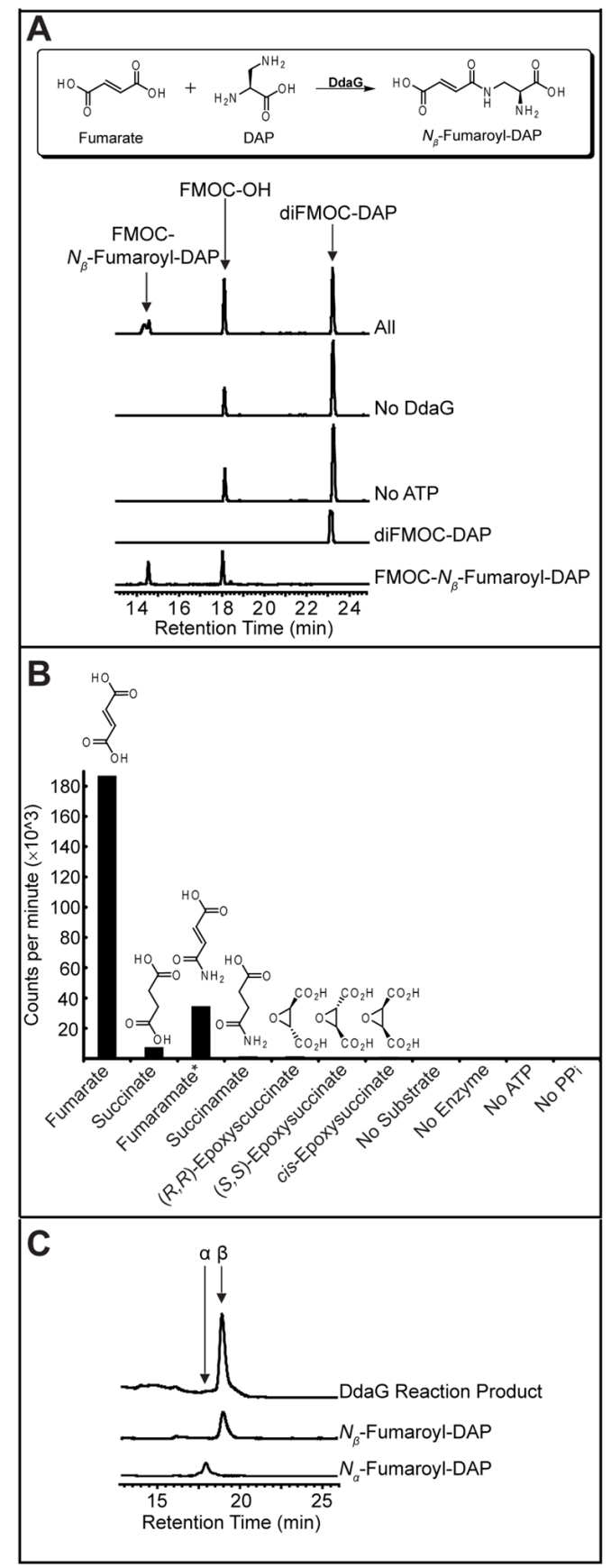

Figure 2.

Characterization of DdaG activity. A) HPLC analysis showing ATP-dependent ligation of fumarate and DAP by DdaG. B) ATP/[ $\left.{ }^{32} \mathrm{P}\right]-\mathrm{PP}_{\mathrm{i}}$ exchange data. Graph shows counts per minute arising from $\left[{ }^{32} \mathrm{P}\right]-\mathrm{ATP}$ that is adsorbed to charcoal after incubation of DdaG, $\left[{ }^{32} \mathrm{P}\right]-\mathrm{PP}$, unlabeled ATP, and carboxylate substrate. *Activity with fumaramate results from 2-5\% contamination with fumarate. ${ }^{2} \mathrm{C}$ ) Coelution of the FMOC-derivatized DdaG product with FMOC- $N_{\beta}$-fumaroyl-DAP. 


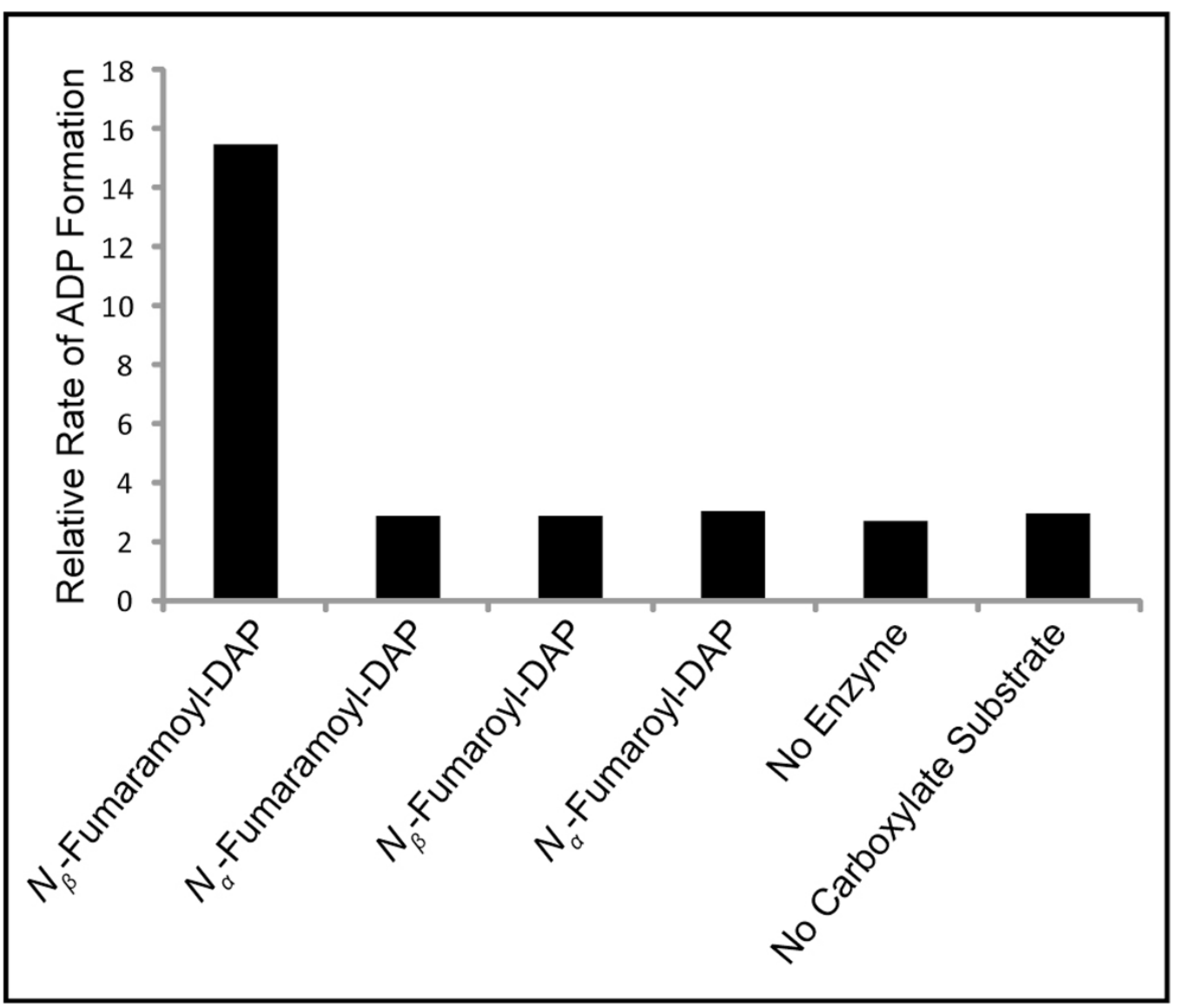

Figure 3.

Relative rates of ADP formation on incubation of DdaF with potential carboxylate substrates and $2 \mathrm{mM}$ Val. 


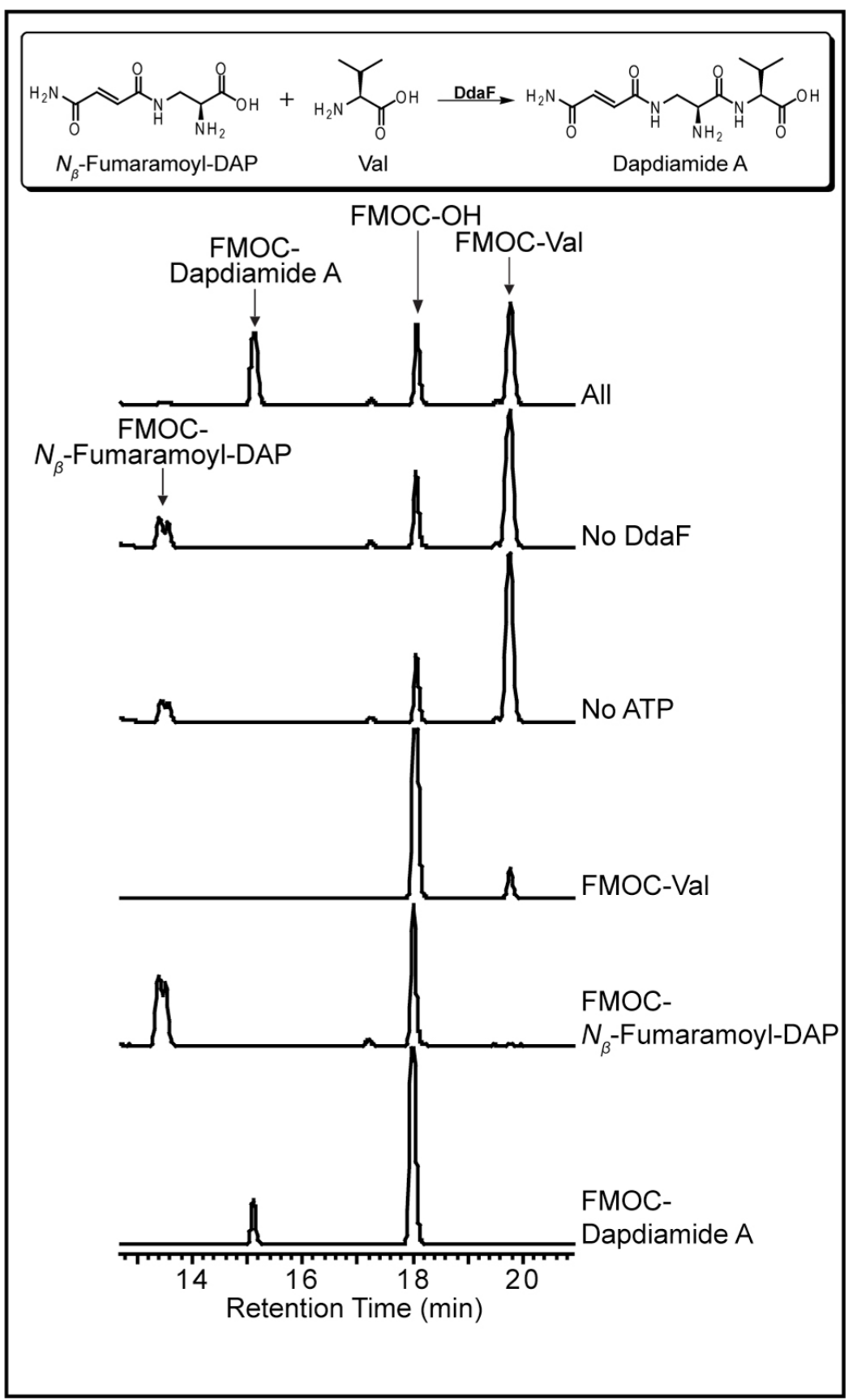

Figure 4.

HPLC analysis showing ATP-dependent ligation of $N_{\beta}$-fumaramoyl-DAP and Val by DdaF to form dapdiamide A. 


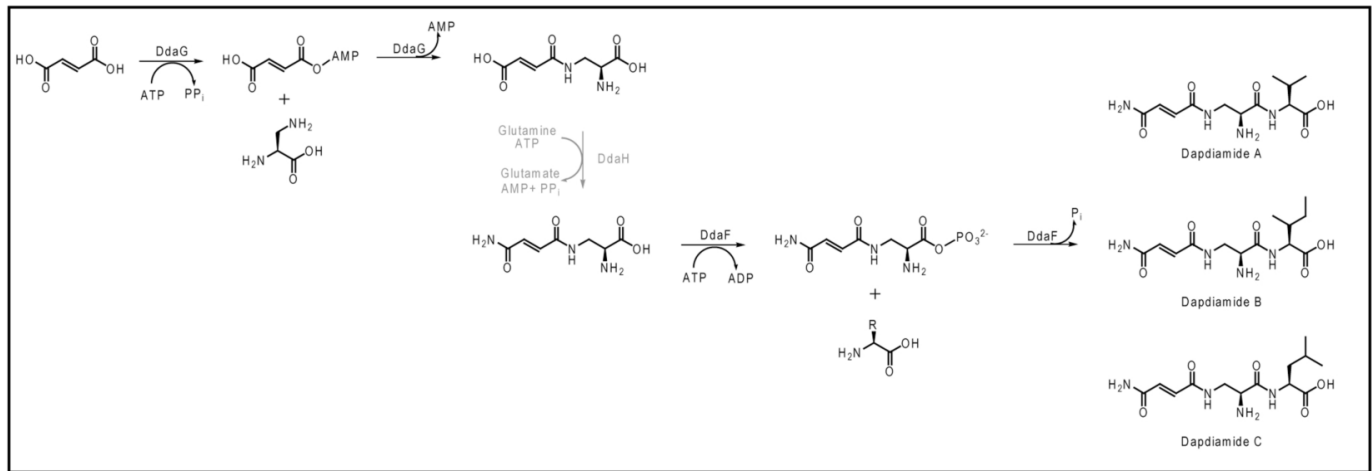

Scheme 1.

Biosynthetic route to dapdiamides A-C. The grayed-out step has not been biochemically validated. 